University of Wollongong

Research Online

Faculty of Engineering and Information

Faculty of Engineering and Information

Sciences - Papers: Part A

Sciences

$1-1-2013$

Performance uniformity evaluation of two SensL's SiPM modules

Lachlan Chartier

University of Wollongong, Ic752@uowmail.edu.au

Yujin Qi

University of Wollongong, yujin@uow.edu.au

Marco Petasecca

University of Wollongong, marcop@uow.edu.au

Peter Ihnat

University of Wollongong, pihnat@uow.edu.au

Michael L. F Lerch

University of Wollongong, mlerch@uow.edu.au

See next page for additional authors

Follow this and additional works at: https://ro.uow.edu.au/eispapers

Part of the Engineering Commons, and the Science and Technology Studies Commons

Research Online is the open access institutional repository for the University of Wollongong. For further information contact the UOW Library: research-pubs@uow.edu.au 


\title{
Performance uniformity evaluation of two SensL's SiPM modules
}

\begin{abstract}
Minimization of the channel-to-channel variation of silicon photomultiplier (SiPM) array is of great importance in achieving high performance for SiPM based imaging detectors. The purpose of this study was to characterize the operating parameters of a large-area SiPM based detector module with $12 \times 12$ pixel array (SensL's ArraySM-4P9) in order to develop an optimal multiplexing readout for high-resolution SPECT imaging. Two versions of SensL's SiPM arrays were investigated in this study. The previous ArraySL-4 version has an array of $4 \times 4$ pixels with $3 \times 3 \mathrm{~mm} 2$ pixel size and the new AarraySM-4p9 version consists of a $3 \times 3$ matrix of the $4 \times 4$ pixels SiPM modules. The current versus voltage (I-V) characteristics of individual SiPM pixels were measured to extract information of its breakdown voltage and dark current. The energy spectrum of individual pixels coupling with a $1 \times 1 \times 3 \mathrm{~mm} 3$ LYSO crystal was measured using $22 \mathrm{Na}$ and $137 \mathrm{Cs}$ sources. The test results show that the previous ArraySL-4 version has larger channel-tochannel variations in breakdown voltage and dark current than the newer AarraySM-4p9 version. The new large-area ArraySM-4P9 SiPM module with $12 \times 12$ pixels shows very small breakdown voltage variations within $\pm 0.1 \mathrm{~V}$ at operating voltage of $\sim 27 \mathrm{~V}$ and dark current variations within $\pm 0.4 \mathrm{nA}$ of $\sim 1 \mathrm{nA}$ over the entire 144 pixel elements. The measured energy resolution of an individual SiPM pixel with a $1 \times 1 \times 3 \mathrm{~mm} 3$ LYSO crystal is $\sim 16 \%$ at energy of $662 \mathrm{keV}$. In conclusion, the new SensL's AarraySM-4p9 ArraySM has much better improved property than the previous ArraySL-4 version. The excellent performance uniformity of the large-area ArraySM-4P9 SiPM module is good for multiplexed readout approach in the development of high-performance and cost-effective compact imaging detectors. $2013 \mathrm{IEEE}$.
\end{abstract}

\section{Keywords}

performance, evaluation, modules, sipm, 039, sensl, uniformity, two

Disciplines

Engineering | Science and Technology Studies

\section{Publication Details}

Chartier, L., Qi, Y., Petasecca, M., Ihnat, P., Lerch, M., Rosenfeld, A. \& Tsui, B. MW. (2013). Performance uniformity evaluation of two SensL's SiPM modules. IEEE Nuclear Science Symposium Conference Record (pp. 1-3). United States: Institute of Electrical and Electronics Engineers.

\section{Authors}

Lachlan Chartier, Yujin Qi, Marco Petasecca, Peter Ihnat, Michael L. F Lerch, Anatoly B. Rosenfeld, and Benjamin Tsui 


\title{
Performance uniformity evaluation of two SensL's SiPM modules
}

\author{
Lachlan Chartier, Yujin Qi, Macro Petasecca, Peter Ihnat, Michael Lerch, Anatoly Rosenfeld, Benjamin MW Tsui
}

\begin{abstract}
Minimization of the channel-to-channel variation of silicon photomultiplier (SiPM) array is of great importance in achieving high performance for SiPM based imaging detectors. The purpose of this study was to characterize the operating parameters of a large-area SiPM based detector module with $12 \times 12$ pixel array (SensL's ArraySM-4P9) in order to develop an optimal multiplexing readout for high-resolution SPECT imaging. Two versions of SensL's SiPM arrays were investigated in this study. The previous ArraySL-4 version has an array of 4x4 pixels with $3 \times 3 \mathrm{~mm}^{2}$ pixel size and the new AarraySM-4p9 version consists of a $3 \times 3$ matrix of the $4 \times 4$ pixels SiPM modules. The current versus voltage (I-V) characteristics of individual SiPM pixels were measured to extract information of its breakdown voltage and dark current. The energy spectrum of individual pixels coupling with a $1 \times 1 \times 3 \mathrm{~mm}^{3}$ LYSO crystal was measured using ${ }^{22} \mathrm{Na}$ and ${ }^{137} \mathrm{Cs}$ sources. The test results show that the previous ArraySL-4 version has larger channel-to-channel variations in breakdown voltage and dark current than the newer AarraySM-4p9 version. The new large-area ArraySM-4P9 SiPM module with $12 \times 12$ pixels shows very small breakdown voltage variations within $\pm 0.1 \mathrm{~V}$ at operating voltage of $\sim 27 \mathrm{~V}$ and dark current variations within $\pm 0.4 \mathrm{nA}$ of $\sim 1 \mathrm{nA}$ over the entire 144 pixel elements. The measured energy resolution of an individual SiPM pixel with a $1 \times 1 \times 3 \mathrm{~mm}^{3}$ LYSO crystal is $\sim 16 \%$ at energy of $662 \mathrm{keV}$. In conclusion, the new SensL's AarraySM-4p9 ArraySM has much better improved property than the previous ArraySL-4 version. The excellent performance uniformity of the large-area ArraySM-4P9 SiPM module is good for multiplexed readout approach in the development of high-performance and costeffective compact imaging detectors.
\end{abstract}

\section{INTRODUCTION}

$\mathrm{I}_{\mathrm{N}}^{\mathrm{N}}$ recent years Silicon Photomultipliers (SiPM) has been aroused considerable attention and interest for applications in nuclear molecular imaging [1-2], because of its appealing properties of great compactness with high gain $\left(\sim 10^{6}\right)$, fast response, low power consumption and insensitive to the magnetic field [3-6]. The feasibility of the SiPM used for the development of high-resolution compact PET and SPECT has been studying by many research groups.

Recent advancements of SiPM array photo-detector technology provide a great opportunity to develop highperformance and cost-effective compact detectors for MRcompatible PET and SPECT systems. However, the design and development of high-resolution detector modules based on the SiPM array into a practical imaging system still present great challenges. Optimizing the coupling between scintillation

Manuscript received November 22, 2013. This work was supported in part by the URC small grant of University of Wollongong.

Lachlan Chartier, Yujin Qi, Macro Petasecca, Michael Lerch, Anatoly Rosenfeld, are with the Centre for Medical Radiation Physics, University of Wollongong, NSW 2522, Australia (e-mail: yujin@uow.edu.au).

crystals and SiPMs, light sharing among elements of the SiPM array, and multiplexed readout schemes are crucial for the detector module to achieve maximal performance.

SensL's ArraySM-4p9 module is a large-area SiPM module with $12 \times 12$ pixels [7]. It's suitable to build the SPECT detector module to provide a relative larger imaging field-ofview. Since the performances of the individual SiPM elements are very sensitive to its operating bias voltage, it's very essential to minimize the channel-to-channel variations of SiPM array in order to develop an optimal multiplexed readout to achieve maximal performance.

In this study, we have evaluated two versions of SensL's SiPM modules of ArraySL-4 and ArraySM-4p9. The current versus voltage (I-V) curve of the individual SiPM pixels was measured. Then the breakdown voltage and dark current of each pixel were extracted. The channel-to-channel variations of individual SiPM pixels in terms of breakdown voltage, dark current, gain and energy resolution would be studied.

\section{MATERIALS AND METHODS}

Two SensL's SiPM array modules of ArraySL-4 and ArraySM-4p9 (shown in Fig. 1) are used in this study. SensL's ArraySL-4 is an old version of small-area SiPM module with $4 \times 4$ pixels while ArraySM-4p9 is a large-area SiPM module which consists of a $3 \times 3$ matrix of SiPM arrays, each with $4 \times 4$ pixels. The active area of ArraySM-4p9 module is $\sim 45 \times 45 \mathrm{~mm}^{2}$. Both modules have a same pixel size of $3 \times 3 \mathrm{~mm}^{2}$, shown in Fig. 1. A special adapt circuit board was made for the ArraySM-4p9 module to lead its 144 pixel signals out for the individual pixel measurements, shown in Fig. 2.

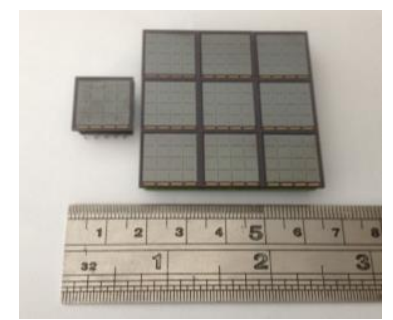

Fig.1 Photo of two SensL's SiPM array modules: ArraySL4 (4x4 pixels) in left and ArraySM-4p9 (12x12 pixels) in right.

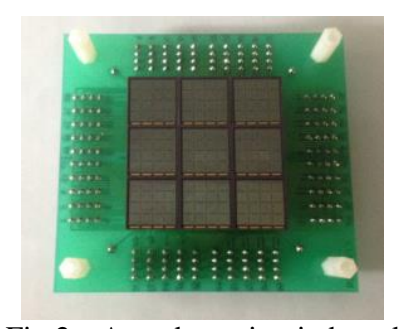

Fig.2 An adapt circuit board made for ArraySM-4p9 module to lead its 144 pixel signals out for individual pixel measurements. 
The current versus voltage (I-V) characteristics of individual SiPM pixel elements were measured using a setup which consists in a Keithly 230 programmable power supply, Keithly 614 electrometer and Keithly 199 multimeter with Labview software control platform. The spectral performances of individual SiPM pixels were evaluated with a $1 \times 1 \times 3 \mathrm{~mm}^{3} \mathrm{LYSO}$ crystal using ${ }^{22} \mathrm{Na}$ and ${ }^{137} \mathrm{Cs}$ sources. The silicone grease was used to couple between the crystal and the SiPM pixel. The energy spectrum of each pixel was measured using the Amptek 8000A MCA.

\section{RESULTS}

\section{A. Results from the ArraySL-4 module}

The measured I-V characteristics of 16 pixels of the ArraySL-4 module are shown in Fig. 3. Four pixels (labeled as B, D, I and K) show significant poor I-V characteristics with large dark current gradients before the breakdown voltages and slow curvatures after the breakdown voltages.

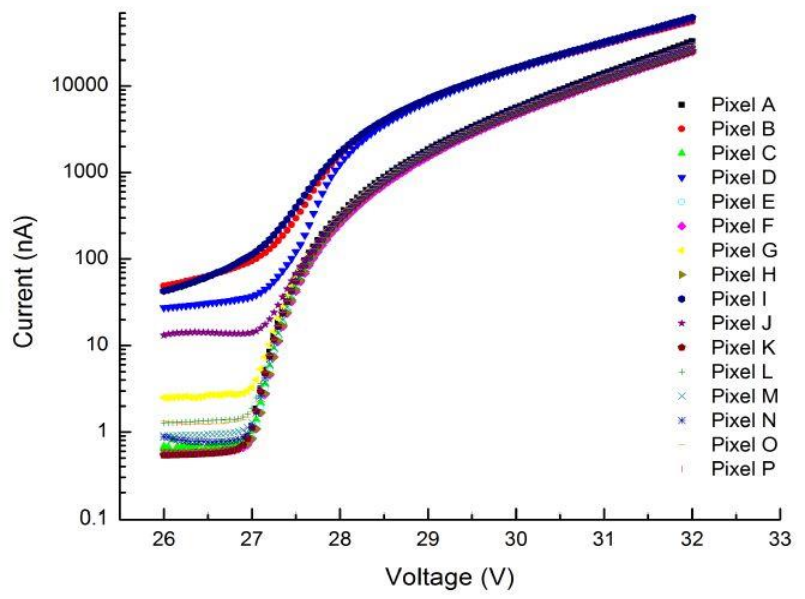

Fig.3 Measured I-V characteristics of all 16 pixels of the ArraySL-4 module.

The extracted breakdown voltage for each pixel is shown in Table 1 . The average breakdown voltage is about $26.9 \mathrm{~V}$ which is slightly lower than the manufacture's value of $27.5 \mathrm{~V}$ at the room temperature.

Table 1: The extracted breakdown voltage for each pixel of ArraySL-4 module

\begin{tabular}{|c|c|c|c|c|c|c|c|c|c|c|c|c|c|c|c|c|}
\hline Pixel label & A & B & C & D & E & F & G & H & I & J & K & L & M & N & O & P \\
\hline $\mathrm{V}_{\text {bd }}(\mathrm{V})$ & 26.80 & 27.00 & 26.90 & 26.90 & 26.75 & 26.75 & 26.85 & 26.85 & 27.00 & 27.05 & 26.90 & 26.85 & 26.85 & 26.85 & 26.90 & 26.80 \\
\hline
\end{tabular}

The spectral performance uniformity of individual pixels has been evaluations with a $1 \times 1 \times 3 \mathrm{~mm}^{3}$ LYSO scintillation crystal using ${ }^{22} \mathrm{Na}$ and ${ }^{137} \mathrm{Cs}$ sources. The measured energy spectra from a typical good SiPM pixel are shown in Fig.3. Then the pixel-to-pixel gain variations can be estimated from the energy photo-peak position variations shown in Fig.4. We estimate that the pixel-to-pixel gain variations are within $\sim 18 \%$ if excluding pixel \#3.

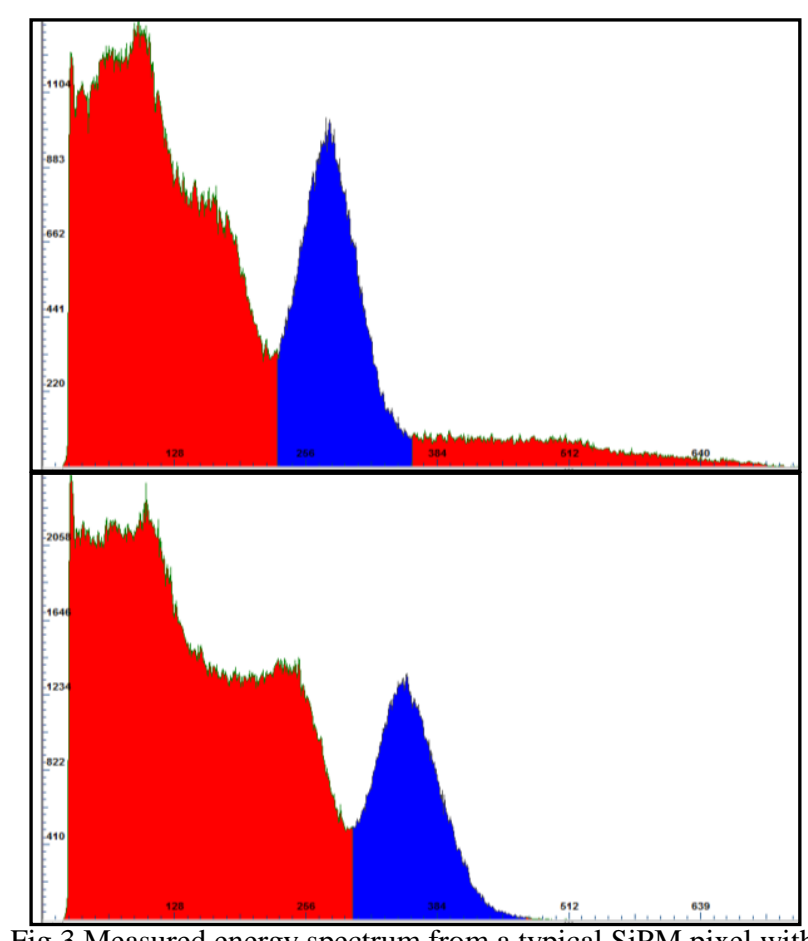

Fig.3 Measured energy spectrum from a typical SiPM pixel with a1 $1 \times 3 \mathrm{~mm}^{3} \mathrm{LYSO}$ crystal using ${ }^{22} \mathrm{Na}$ (top) and ${ }^{137} \mathrm{Cs}$ (bottom) source at hias voltage of $30 \mathrm{~V}$.

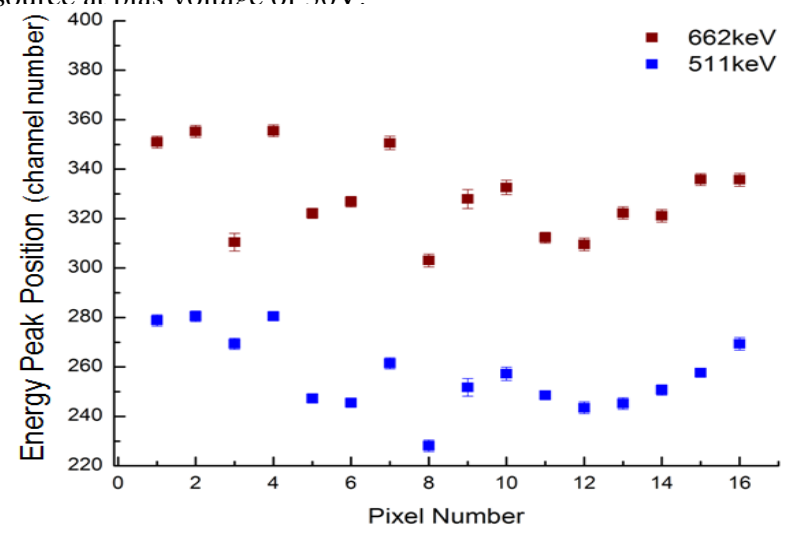

(a)

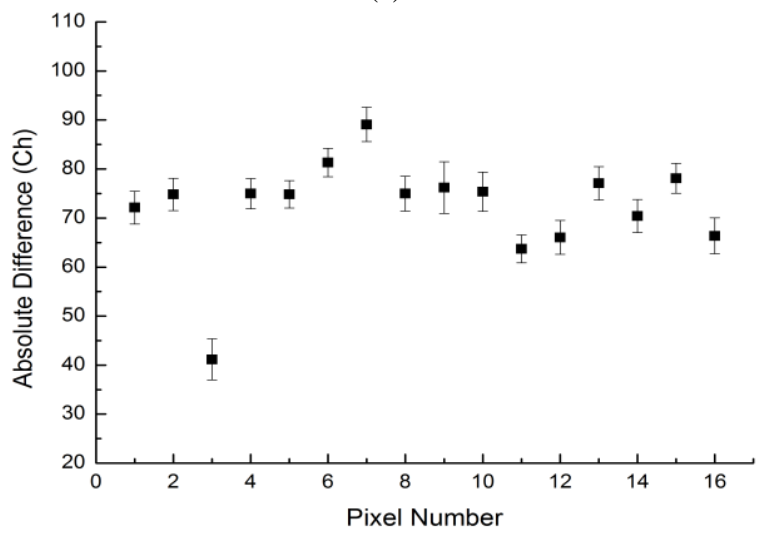

(b)

Fig.4 Measured pixel-to-pixel variation of ArraySL-4 with a $1 \times 1 \times 3 \mathrm{~mm}^{3}$ LYSO crystal at bias voltage of $30 \mathrm{~V}$ using ${ }^{22} \mathrm{Na}$ and ${ }^{137} \mathrm{Cs}$ sources. (a): energy peak position variations; (b): pixel-topixel variation of the peak-position difference between $662 \mathrm{keV}$ and $511 \mathrm{keV}$. 
The energy resolutions from individual pixels are also measured, shown in Fig. 5. The average energy resolutions are $\sim 16 \%$ at gamma-rays energy of $662 \mathrm{keV}$ and $\sim 19 \%$ at energy of $511 \mathrm{keV}$ as comparing to the measured energy resolution of $\sim 13.6 \%$ with a standard PMT at $511 \mathrm{keV}$.

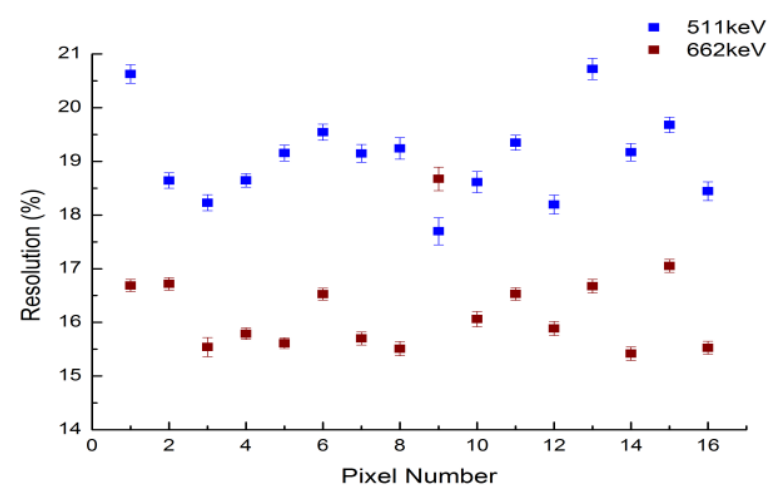

Fig.5 Extracted pixel-to-pixel variations of energy resolutions with gamma-ray energies of $511 \mathrm{kev}$ and $662 \mathrm{keV}$.

\section{B. Results from the ArraySM-4p9 module}

The measured I-V characteristics of ArraySM-4p9 module are shown in Fig. 6. All the 144 pixels show the consistent I-V characteristics. There is no particular poor pixel in this module. The channel-to-channel variation in breakdown voltages is shown in Fig.6. It shows very small variations of $\pm 0.1 \mathrm{~V}$ around the nominal $27 \mathrm{~V}$ within the measurement error of $\pm 0.1 \mathrm{~V}$. The

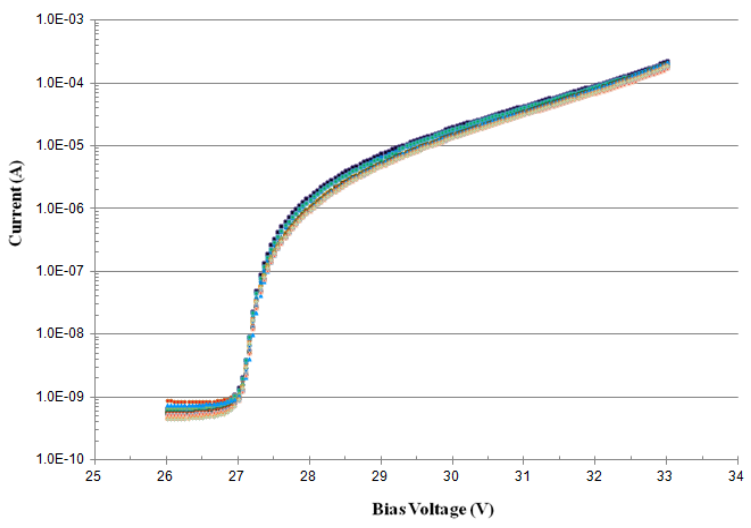

Fig.5 Measured I-V characteristics of 16 pixels from one of the $3 \times 3$ units of the ArraySM-4p9 module.

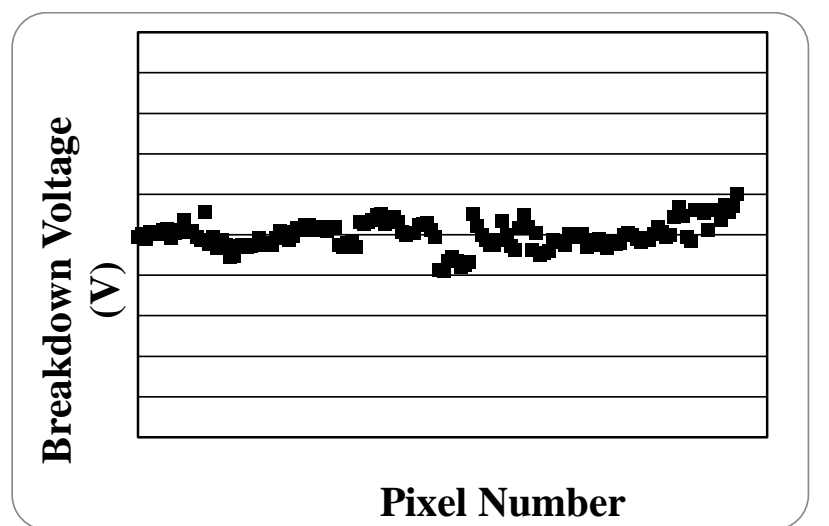

Fig.6 Extracted breakdown voltage pixel-to-pixel variations of ArraySM-4p9 over all the 144 pixels. channel-to-channel variation in average dark currents is shown in Fig.7. It shows a small variation of $\pm 0.4 \mathrm{nA}$ around $1 \mathrm{nA}$. These indicate that the ArraySM-4p9 module has very good uniformity over its 144 pixels

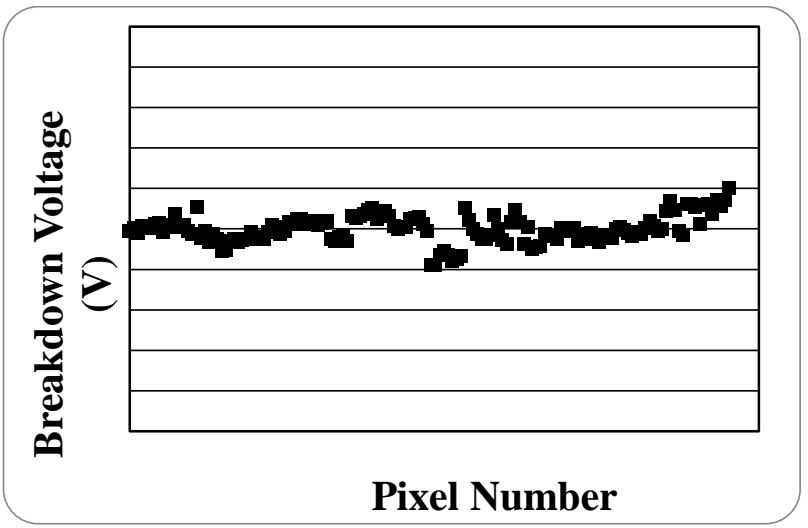

Fig.7 Extracted pixel-to-pixel variations of dark current from the ArraySM-4p9 module over all the 144 pixels.

\section{CONCLUSION AND DISCUSSION}

We have evaluated the performance of two SiPM array modules from the SensL's ArraySL and ArraySM series. The preliminary results show that SensL's ArraySM series has much better performance property than its old version of ArraySL series. The large-area ArraySM-4p9 module shows excellent performance uniformity which is very good for multiplexing readout approach to develop high-performance and cost-effective compact imaging detectors. The spectral performances of the ArraySM-4p9 module are still undergoing and then the multiplexing readout for it will be developed for MR-compatible SPECT detector module.

\section{REFERENCES}

[1] Renker, D., and E. Lorenz. "Advances in Solid State Photon Detectors." Journal of Instrumentation 4(4):P04004, 2009.

[2] Ronicali E, Cherry S.R, "Application of Silicon Photomultiplier to Positron Emission Tomography," Ann Biomed Eng, 39(4):1358-1377, 2011.

[3] D. Renker, "Geiger-mode avalanche photodiodes, history, properties and problems," Nucl. Instr. Meth. Phys. Res., vol. 567, pp. 48-56, Jun. 2006.

[4] G. Llosa, N. Belcari, M. G. Bisogni, G. Collazuol, S. Marcatili, P. Barrillon, et al., "Energy, timing and position resolution studies with 16pixel silicon photomultiplier matrices for small animal PET," IEEE Trans. Nucl. Sci., vol. 56, no. 56, pp. 2586-2593, Oct. 2009.

[5] T. Y. Song, H. Wu, S. Komarov, S. B. Siegel, Y.-C. Tai, "A submillimeter resolution PET detector module using a multi-pixel photon counter array," Phys. Med. Biol., Vol. 55, pp. 2573-2587, 2010.

[6] D. R. Schaart, H. T. van Dam, S. Seifert, R. Vinke, P. Dendooven, H. Lohner, and F. J. Beekman, "A novel, SiPM-array-based, monolithic scintillator detector for PET," Phys. Med. Biol. vol. 54, pp. 3501-3512, 2009.

[7] Sensl, http://sensl.com/products/silicon-photomultipliers/arraysm4p9/. 